\title{
Study on Bioefficacy of Insecticides against Shoot Weevil (Alcidodes affaber Aurivillius) in Okra (Abelmoschus esculentus Linn.)
}

\author{
H. A. Manjunatha ${ }^{1 *}$, S. B. Patil ${ }^{2}$, S. S. Udikeri ${ }^{3}$ and Shamarao Jahagirdhar ${ }^{4}$ \\ ${ }^{1}$ Department of Agricultural Entomology, UAS, Dharwad - 580 005, Karnataka, India \\ ${ }^{2}$ Associate Director of Extension, Vijayapura, UAS, Dharwad - 580 005, Karnataka, India \\ ${ }^{3}$ Cotton Entomology, AICCIP, ARS, Heballi, UAS, Dharwad - 580 005, Karnataka, India \\ ${ }^{4}$ Plant Pathology, Soybean Scheme, MARS, UAS, Dharwad - 580 005, Karnataka, India \\ *Corresponding author
}

\begin{tabular}{|c|c|}
\hline & A B S T R A C T \\
\hline $\begin{array}{l}\text { K e y w o r d s } \\
\text { Bioefficacy, } \\
\text { Insecticides, Okra, } \\
\text { Shoot weevil } \\
\text { (Alcidodes affaber), } \\
\text { Yield. }\end{array}$ & \multirow{3}{*}{$\begin{array}{l}\text { Experiment was conducted during } 2015 \text { kharif season at MARS, Dharwad to investigate } \\
\text { the bioefficacy of insecticides against shoot weevil Alcidodes affaber in okra by using a } \\
\text { variety Arka anamika. Randomized block design was followed with } 9 \text { treatments and } \\
\text { replicated thrice. The results indicated that application of } \\
\text { Profenophos50EC@2mL/L+DDVP 76EC@0.5mL/L at } 20,40 \text { and } 60 \text { DAS was effective } \\
\text { in reducing the incidence of shoot weevil and recorded significantly higher okra fruit yield } \\
\text { of } 8316 \text { kg/ha, followed by treatment sprayed with } \\
\text { Thiodicarb70WP@ } 1.0 \mathrm{~g} / \mathrm{L}+\mathrm{DDVP} 76 \mathrm{EC} @ 0.5 \mathrm{~mL} / \mathrm{L}(6850.13 \mathrm{~kg} / \mathrm{ha}) \text {. On the contrary } \\
\text { untreated check yielded significantly lowest yield of } 1264.28 \mathrm{~kg} / \mathrm{ha} \text {. Field observations } \\
\text { suggested that sparaying of Profenophos } 50 \mathrm{EC} @ 2 \mathrm{~mL} / \mathrm{L}+\mathrm{DDVP} 76 \mathrm{EC} @ 0.5 \mathrm{~mL} / \mathrm{L} \text { was } \\
\text { economical and most remunerative recorded highest benefit cost ratio (B:C ratio) of } 3.83 \text { it } \\
\text { was followed by Thiodicarb 70WP@ } 1.0 \mathrm{~g} / \mathrm{L}+\mathrm{DDVP} 76 \mathrm{EC} @ 0.5 \mathrm{~mL} / \mathrm{L} \text {. }\end{array}$} \\
\hline Article Info & \\
\hline $\begin{array}{l}\text { Accepted: } \\
\text { 10 July } 2017 \\
\text { Available Online: } \\
\text { 10 September } 2017\end{array}$ & \\
\hline
\end{tabular}

\section{Introduction}

Okra (Abelmoschus esculentus L. Moench), known as lady's finger or bhendi, belongs to family Malvaceae and is an important vegetable crop grown throughout the year. It's a native of tropical Africa. Besides India, it is being grown in many tropical and subtropical parts of the world. The world total area and production under okra is reported to be around 1148.0 thousand ha and 7896.3 thousand tons. India stands first in world with an area of 0.5 million ha, with a production of 5.79 metric million tons and productivity of $11.9 \mathrm{MT} / \mathrm{ha}$ followed by Nigeria. Okra contributes 3.9 per cent share in total vegetable production of India and 72.9 per cent of its share in world vegetable production, Within India, West Bengal stands first with respect to area and production and highest productivity is reported from Chattisgarh (Anon., 2015). One of the important limiting factors in the cultivation of okra is ravages caused by insect pests. As high as 72 species of insects have been recorded on okra (Srinivas Rao and Rajendran, 2003) of which Weevils have been found to be infesting a variety of host plants both in the field as well as in the storage. In India, four species of shoot weevil viz., 
Alcidodes affaber Aurivillius, Alcidodes leopardus olivier, Alcidodes fabricii Fabricius and Alcidodes mysticus (Faust) have been found to infest cotton. Among these Alcidodes affaber Aurivillius was first noted and described by Aurivillius in 1891, it was reported from many parts of the country. Further Ayyar (1922), reported okra (Abelmoschus esculentus L. Moench), as a most prefered host plant for shoot weevil.

Shoot weevil causes considerable damage to the crop and assuming a major pest status as its population reaches a high during June to September. In North Western Karnataka, particularly in the transitional zone of Dharwad, Belgaum and Uttar Kannada districts, the shoot weevil A. affaber has been causing considerable damage (Deshpande, 1990). Meagre information on appropriate management practice with newer molecules and insecticide combi products with suitable spray schedule for its effective management in okra. Its menace is increasing year after year particularly in transgenic Bt-cotton and Bhendi growing areas of Karnataka and Tamilnadu, posing challenges to okra cultivation. Hence, the present investigation was undertaken to assess the efficacy of insecticides against shoot weevil in okra for its effective management.

\section{Materials and Methods}

The field trial was conducted at MARS, Dharwad during 2015 kharif seasons under rainfed conditions. The okra variety 'Arka anamika' was raised in plots with a plot size involved five rows of 3.6 meters length and twelve plants in a row of 3.0 meters wide with a $60 \times 30 \mathrm{~cm}$ between row to row and plant to plant spacing. Each plot accommodated 60 plants. All the recommended agronomic practices were followed except plant protection measures. The experiment was laid out in randomized block design with nine treatments consists of spraying the crop with
Profenophos 50EC@2mL/L, Novaluron 10EC@1.0mL/L, Azadirichtin 1500ppm @ 5mL/L, Thiodicarb 70WP@1.0g/ml, Profenophos 50EC@2mL/L+ DDVP 76EC $@ 0.5 \mathrm{~mL} / \mathrm{L}$, Novaluron10EC @ $1.0 \mathrm{~mL} / \mathrm{L}+$ DDVP76 EC@0.5 mL/L, Azadirichtin 1500ppm @ $5 \mathrm{~mL} / \mathrm{L}+$ DDVP 76EC @ 0.5 mL/L, Thiodicarb 70WP @ 1g/L + DDVP 76EC @ $0.5 \mathrm{~mL} / \mathrm{L}$ including untreated control and replicated three times. Totally three sprays were taken at 20,40 and 60 days after sowing (DAS) in all the treatments. Application of chemical insecticides was taken up by using high volume knapsack sprayer at the rate of 500 literes of spray fluid per hectare.

For assessing the efficacy of newer molecules, the observations were recorded on 10 randomly selected plants per treatment. Observations recorded were number of adult weevils per plot, during morning hours (8 AM) in each treatment. Further, per cent reduction over control was calculated by using the formula,

(Per cent infestation in UTC Per cent infestation in treated treatment) $\%$ reduction over control = ------------- x 100

Per cent infestation in UTC

Where, UTC- Untreated control

For recording the grub load, tunnel length and number of broken branches, destructive sampling was carried out in ten randomly selected infested plants at seventy days after the sowing. During the observation, the stem of each infested plant was split open to record number of grubs, number of broken branches and tunnel length. Finally tunnel length was measured in terms of centimeter.

For recording fruit yield, matured medium sized tender okra fruits were harvested separately from each plot at three to four days interval for six weeks after the 50 days of 
crop age and confirmed upto end of cropping period. Fruit yield obtained from each plot (kg per plot) was expressed in terms yield per hectare (kg per ha). Finally the per cent increase in yield for each treatment was calculated over control using the following formula,

(Yield in chemically protected plot Yield in untreated treatment) Per cent increase in yield $=$------------- $\times 100$

Yield in chemically protected plot

The data on incidence of shoot weevil and yields were subjected to suitable statistical analysis. The mean values of the treatments were statistically analyzed and then separated by Duncan's Multiple Range Test (DMRT). Based on the yield data, cost of plant protection and total cost of production, the gross returns and net returns were calculated for each treatment. The gross returns were worked out by taking the selling price of okra fruits and the net returns of different treatments were worked out by deducting total cost of production from gross returns. Finally, B: C ratio was worked out by dividing gross returns and total cost of production in each treatment.

\section{Results and Discussion}

The results with respect to adult shoot weevil population (Table 1), grub load, tunnel length, broken branches, number of galls on plants (Table 2) and okra fruit yield (Table 3) were quite significant, indicating the differential impact of chemical intervention.

The pooled analysis of three sprays indicated that, Profenophos 50 EC @ 2 mL/L + DDVP $76 \mathrm{EC} @ 0.5 \mathrm{~mL} / \mathrm{L}$ was found to be significantly superior by recording the least population and higher per cent reduction (7.82/plot and $63.20 \%$ ) of shoot weevil adult population among the treatments followed by
Thiodicarb 70WP @ 1g/L + DDVP 76EC @ $0.5 \mathrm{~mL} / \mathrm{L}$ (9.35/plot and 55.99\%) and Novaluron 10EC @ 1.0 mL/L + DDVP 76EC @ $0.5 \mathrm{~mL} / \mathrm{L}$ (9.63/plot and $54.65 \%)$ and were at par with each other.

However, Nimbicidin 1500ppm + DDVP 76EC @ $5 \mathrm{~mL} / \mathrm{L}+0.5 \mathrm{~mL} / \mathrm{L}$ (10.17/plot and $52.14 \%$ ) and profenophos 50 EC@2 mL/L (11.77/plot and $44.61 \%)$ were found to be next best treatments. On the contrary, the population of shoot weevil was continued to be on higher side in untreated control (21.24/plot). Similar trend with respect to population reduction was observed even after the subsequent sprays (Table 1).

Observation on number of grubs per plant, tunnel length and number of broken branches in each treatment was recorded to evaluate the efficacy of different treatments. The observations recorded on number of grubs per plant revealed that, the application of Profenophos 50 EC @ $2 \mathrm{~mL} / \mathrm{L}+$ DDVP 76EC @ $0.5 \mathrm{~mL} / \mathrm{L}$ recorded least number of grubs per plant (0.87/plant) and was statistically superior over rest of the treatments. Thiodicarb 70WP @ 1.0g/L + DDVP 76EC @ $0.5 \mathrm{~mL} / \mathrm{L}$ (1.80/plot) was found to be next best treatment. While significantly higher number of grubs per plant was recorded in rest of all other treatments including in untreated control. Stem tunnel length caused due to infestation by shoot weevil was taken as criteria to assess the efficacy of the treatments. Among the treatments, lowest tunnel length of $7.30 \mathrm{~cm}$ was recorded in Profenophos 50 EC @ 2 mL/L + DDVP 76EC @ $0.5 \mathrm{~mL} / \mathrm{L}$ treatment followed by Thiodicarb 70WP @ 1.0g/L + DDVP 76EC @ $0.5 \mathrm{~mL} / \mathrm{L}(8.73 \mathrm{~cm})$ and Novaluron $10 \mathrm{EC}$ @ $1 \mathrm{~mL} / \mathrm{L}+$ DDVP 76EC @ $0.5 \mathrm{~mL} / \mathrm{L}$ $(9.90 \mathrm{~cm})$. Significantly highest tunnel length of $15.65 \mathrm{~cm}$ and $23.87 \mathrm{~cm}$ was recorded in Nimbicidin 1500ppm @ $5 \mathrm{~mL} / \mathrm{L}$ and untreated control, respectively (Table 2). 
Observations recorded on number of broken branches due to shoot weevil infestation per ten infested plants revealed that, Profenophos 50 EC@ 2 mL/L + DDVP 76 EC @ 0.5 $\mathrm{mL} / \mathrm{L}$ recorded significantly least number of broken branches (1/10 infested plants) and was significantly superior over other treatments followed by Thiodicarb 70WP @ 1.0g/L + DDVP 76 EC @ 0.5 mL/L (2.1/10 infested plants).

On the contrary, highest number of broken branches per 10 infested plants was observed in rest of the treatments. Observations recorded on number of galls due to weevil infestation per ten infested plants revealed that, Profenophos 50 EC @ 2 mL/L + DDVP 76 EC @ $0.5 \mathrm{~mL} / \mathrm{L}$ recorded significantly least number of galls (1.23/10 infested plants) and was significantly superior over other treatments followed by Thiodicarb 70WP @ 1.0g/L + DDVP 76 EC @ 0.5 mL/L (2/10 infested plants) and found next best treatment with respect to number of galls caused by shoot weevil infestation. On the contrary, highest number of galls 7.5 and 8.63 was observed in Nimbicidin 1500ppm @ 5 mL/L and untreated control, respectively (Table 3).
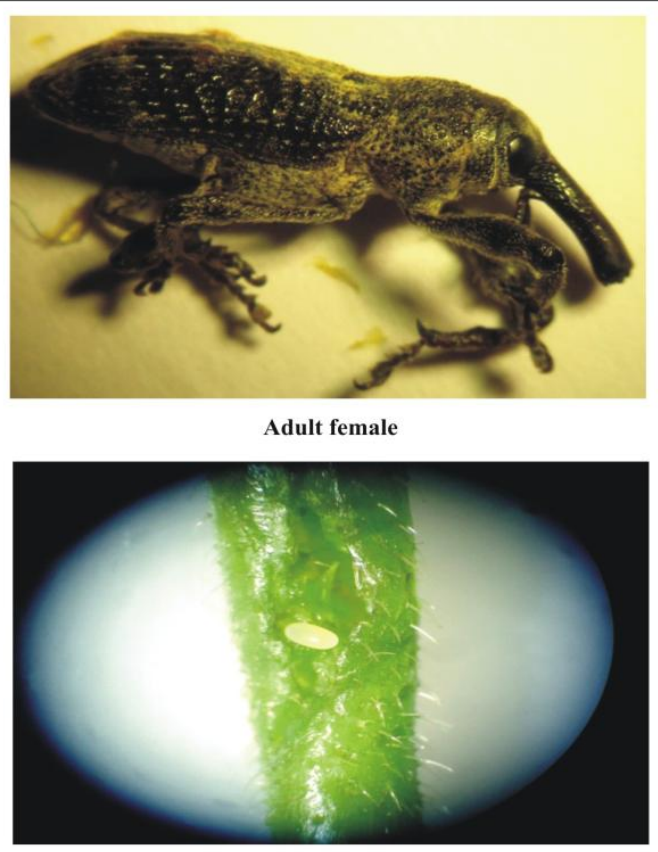

Shoot weevil egg

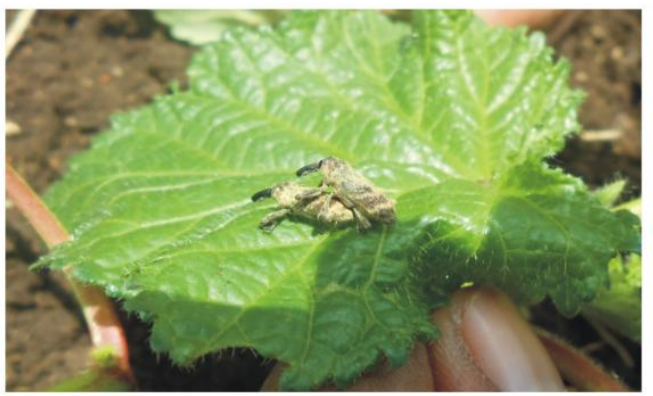

Shoot weevil adults in mating process

Plate 10: Adult activity 


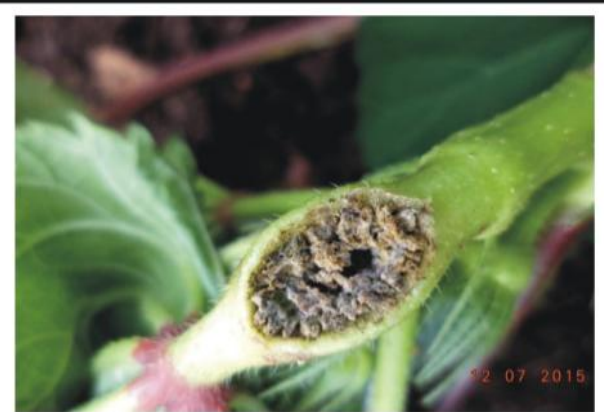

Galls with excreta

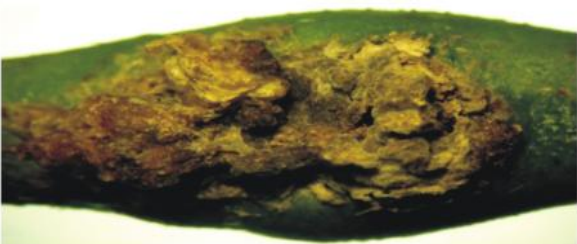

Grub entry site and stem galls at later stages of plants

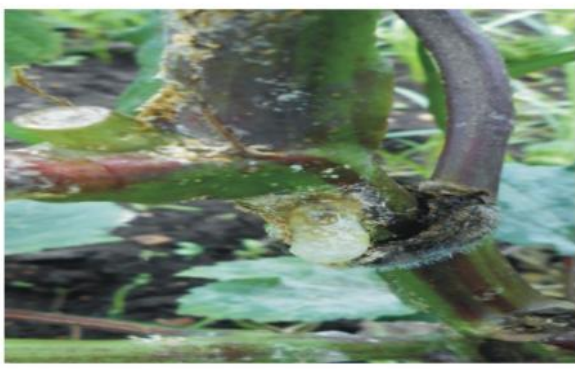

Oozing of plant sap (frothy secretion) from grub entry hole on main stem

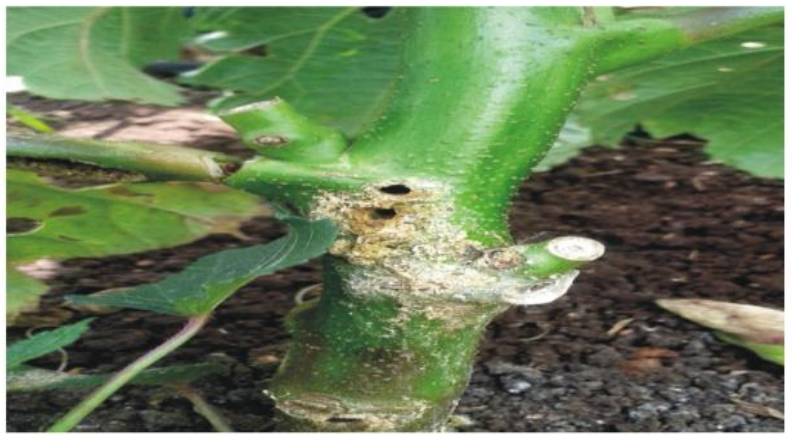

Adult emerged holes after complete development from immature stages inside the stem

Plate 12: Damaging symptoms caused by shoot weevil at grub stage

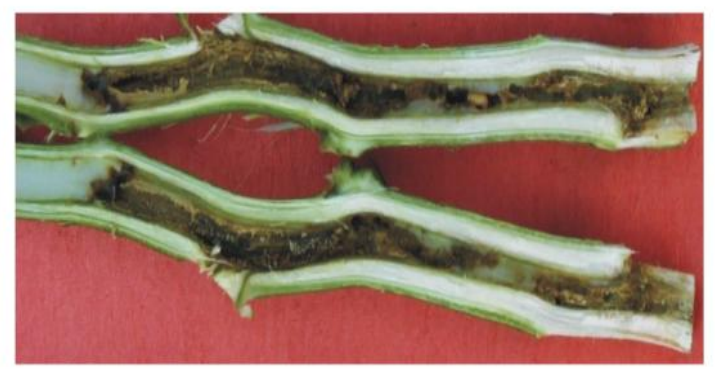

An average of $23 \mathrm{~cm}$ tunnel length was noticed in crop left for natural infestation

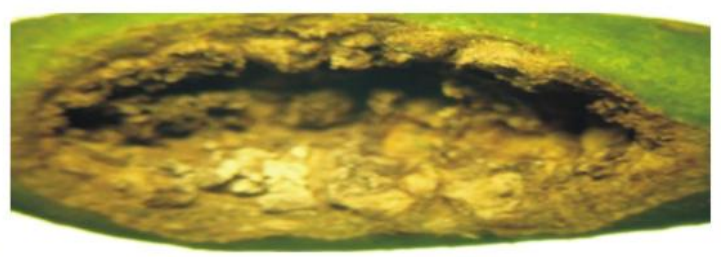

Galls on side branches and main stem 
Table.1 Bio efficacy of insecticides against shoot weevil population in okra

\begin{tabular}{|c|c|c|c|c|c|c|c|c|c|c|c|c|c|c|c|c|c|c|c|c|}
\hline \multirow{3}{*}{$\begin{array}{c}\text { Treatment } \\
\text { number }\end{array}$} & \multicolumn{18}{|c|}{ Shoot weevil adult population per plot } & \multirow{2}{*}{\multicolumn{2}{|c|}{$\begin{array}{c}\text { Pooled data of } \\
3 \text { sprays }\end{array}$}} \\
\hline & \multicolumn{6}{|c|}{$1^{\text {st }}$ Spray (20 DAS) } & \multicolumn{6}{|c|}{$2^{\text {nd }}$ Spray (40 DAS) } & \multicolumn{6}{|c|}{$3^{\text {rd }}$ spray (60 DAS) } & & \\
\hline & BS & 5 DAS & 10 DAS & 15 DAS & Mean $\mathbf{F}$ & POC & BS & 5 DAS & 10 DAS 1 & 15 DAS & Mean & POC & BS & 5 DAS & 10 DAS & 15 DAS & Mean & POC & $\begin{array}{l}\text { Total } \\
\text { Mean }\end{array}$ & POC \\
\hline $\mathrm{T}_{1}$ & $\begin{array}{l}16.79 \\
(4.13)\end{array}$ & $\mid \begin{array}{c}14.59 \\
(3.88) \mathrm{ab}\end{array}$ & $\mid \begin{array}{c}13.80 \\
(3.78) \mathrm{abc}\end{array}$ & $\mid \begin{array}{c}13.35 \\
(3.72) \mathrm{ab}\end{array}$ & $\begin{array}{c}14.63 \\
(3.87) \mathrm{b}\end{array}$ & 24.97 & $\begin{array}{c}12.11 \\
(3.55) \mathrm{bc}\end{array}$ & $\mid \begin{array}{c}11.90 \\
(3.52) \mathrm{bc}\end{array}$ & $\begin{array}{c}11.87 \\
(3.52) \mathrm{cde}\end{array}$ & $\mid \begin{array}{c}10.71 \\
(3.35) b c\end{array}$ & $\mid \begin{array}{c}11.65 \\
(3.49) \mathrm{bc}\end{array}$ & 46.03 & $\begin{array}{c}10.44 \\
(3.3) \mathrm{bcd}\end{array}$ & $\begin{array}{c}9.18 \\
(3.11) \mathrm{bc}\end{array}$ & $\begin{array}{c}8.65 \\
(3.02) \mathrm{bcd}\end{array}$ & \begin{tabular}{|c|}
7.83 \\
$(2.88) \mathrm{c}$
\end{tabular} & $\begin{array}{c}9.03 \\
(3.08) \mathrm{bcd}\end{array}$ & 60.16 & $\begin{array}{c}11.77 \\
(3.49) \mathrm{bcd}\end{array}$ & 44.61 \\
\hline $\mathrm{T}_{2}$ & $\begin{array}{l}17.35 \\
(4.22)\end{array}$ & $\mid \begin{array}{c}16.14 \\
(4.08) \mathrm{ab}\end{array}$ & $\begin{array}{c}15.48 \\
(3.99) \mathrm{bc}\end{array}$ & \begin{tabular}{c|}
14.76 \\
$(3.9) \mathrm{b}$
\end{tabular} & $\begin{array}{c}15.93 \\
(4.05) \mathrm{b}\end{array} \mid 1$ & 18.29 & \begin{tabular}{|c|}
13.00 \\
$(3.67) \mathrm{c}$
\end{tabular} & $\mid \begin{array}{c}12.91 \\
(3.65) \mathrm{bc}\end{array}$ & $\begin{array}{c}12.29 \\
(3.57) \mathrm{de}\end{array}$ & & $\mid \begin{array}{c}12.62 \\
(3.62) \mathrm{c}\end{array}$ & 41.56 & $\begin{array}{c}12.47 \\
(3.6) \mathrm{cd}\end{array}$ & & $\begin{array}{c}10.91 \\
\text { (3.35)de }\end{array}$ & \begin{tabular}{|c|}
10.00 \\
$(3.24) \mathrm{d}$
\end{tabular} & $\begin{array}{c}11.28 \\
(3.43) \mathrm{de}\end{array}$ & 50.20 & $\mid \begin{array}{c}13.28 \\
(3.7) \mathrm{bcd}\end{array}$ & 37.51 \\
\hline $\mathrm{T}_{4}$ & $\begin{array}{l}18.51 \\
(4.36)\end{array}$ & $\begin{array}{c}15.04 \\
(3.9) \mathrm{ab}\end{array}$ & \begin{tabular}{|c|}
14.01 \\
$(3.81) \mathrm{bc}$
\end{tabular} & \begin{tabular}{|c|}
13.98 \\
$(3.79) \mathrm{b}$
\end{tabular} & $\left.\begin{array}{c}15.39 \\
(3.96) \mathrm{b}\end{array}\right|^{2}$ & 21.09 & $\begin{array}{c}12.96 \\
(3.63) \mathrm{bc}\end{array}$ & $\begin{array}{c}12.15 \\
(3.56) \mathrm{bc}\end{array}$ & $\mid \begin{array}{c}11.24 \\
(3.42) \mathrm{bcd}\end{array}$ & \begin{tabular}{|c|}
10.93 \\
$(3.38) \mathrm{c}$ \\
\end{tabular} & $\left|\begin{array}{c}11.82 \\
(3.51) \mathrm{bc}\end{array}\right|$ & 45.23 & $\begin{array}{c}10.82 \\
(3.36) \mathrm{cd}\end{array}$ & $\begin{array}{c}10.02 \\
(3.23) \mathrm{cd}\end{array}$ & $\begin{array}{c}9.6 \\
(3.18) \mathrm{cde}\end{array}$ & \begin{tabular}{|c|}
9.35 \\
$(3.13) \mathrm{d}$
\end{tabular} & $\begin{array}{c}9.95 \\
(3.23) \mathrm{cd}\end{array}$ & 56.08 & $\mid \begin{array}{c}12.39 \\
(3.58) \mathrm{bcd}\end{array}$ & 41.70 \\
\hline $\mathrm{T}_{5}$ & $\begin{array}{l}.03 \\
.06)\end{array}$ & $\begin{array}{c}11.49 \\
(3.46) \mathrm{a}\end{array}$ & \begin{tabular}{|c|}
9.93 \\
$(3.21) \mathrm{a}$
\end{tabular} & $\begin{array}{c}9.00 \\
(3.07) \mathrm{a}\end{array}$ & \begin{tabular}{c|c}
11.61 \\
$(3.48) \mathrm{a}$
\end{tabular} & 40.44 & \begin{tabular}{|c|}
8.76 \\
$(3.04) \mathrm{a}$
\end{tabular} & \begin{tabular}{|c|}
8.22 \\
$(2.95) \mathrm{a}$
\end{tabular} & \begin{tabular}{|c|}
8.13 \\
$(2.93) \mathrm{a}$
\end{tabular} & \begin{tabular}{|c|}
8.02 \\
$(2.92) \mathrm{a}$
\end{tabular} & \begin{tabular}{|c|}
8.28 \\
$(2.96) \mathrm{a}$
\end{tabular} & 61.64 & \begin{tabular}{|c|}
6.45 \\
$(2.64) \mathrm{a}$
\end{tabular} & $\begin{array}{c}5.13 \\
(2.35) \mathrm{a}\end{array}$ & $\begin{array}{c}1.99 \\
(1.56) \mathrm{a}\end{array}$ & $\begin{array}{c}0.65 \\
(1.07) \mathrm{a}\end{array}$ & $\begin{array}{c}3.56 \\
(2.01) \mathrm{a}\end{array}$ & 84.29 & $\begin{array}{c}7.82 \\
(2.82) \mathrm{a}\end{array}$ & 63.20 \\
\hline $\mathrm{T}_{6}$ & $\begin{array}{l}18.73 \\
(4.37)\end{array}$ & $\mid \begin{array}{c}14.71 \\
(3.89) \mathrm{ab}\end{array}$ & $\begin{array}{c}11.44 \\
(3.45) \mathrm{ab}\end{array}$ & $\begin{array}{c}9.78 \\
(3.2) \mathrm{ab}\end{array}$ & $\left.\begin{array}{c}13.66 \\
(3.76) \mathrm{ab}\end{array}\right|^{2}$ & 29.93 & $\begin{array}{c}9.66 \\
(3.17) \mathrm{bc}\end{array}$ & \begin{tabular}{|c|}
9.35 \\
$(3.12) \mathrm{bc}$
\end{tabular} & $\begin{array}{c}9.16 \\
(3.1) \mathrm{bc}\end{array}$ & $\begin{array}{c}8.33 \\
(2.96) \mathrm{b}\end{array}$ & $\mid \begin{array}{c}9.12 \\
(3.1) \mathrm{bc}\end{array}$ & 57.73 & $\begin{array}{c}7.79 \\
(2.88) \mathrm{b}\end{array}$ & \begin{tabular}{|c|}
7.61 \\
$(2.85) \mathrm{bc}$
\end{tabular} & $\begin{array}{c}5.22 \\
(2.39) \mathrm{b}\end{array}$ & $\begin{array}{c}3.85 \\
(2.06) \mathrm{bc}\end{array}$ & $\begin{array}{c}6.12 \\
(2.57) \mathrm{b}\end{array}$ & 73.00 & $\begin{array}{c}9.63 \\
(3.15) \mathrm{bc}\end{array}$ & 54.65 \\
\hline $\mathrm{T}_{7}$ & $\begin{array}{l}18.33 \\
(4.31)\end{array}$ & $\mid \begin{array}{c}15.35 \\
(3.98) \mathrm{ab}\end{array}$ & \begin{tabular}{|c|}
11.81 \\
$(3.51) \mathrm{abc}$
\end{tabular} & $\begin{array}{c}10.55 \\
(3.31) \mathrm{ab}\end{array}$ & $\begin{array}{c}14.01 \\
(3.81) \mathrm{ab}\end{array} \mid 2$ & 28.14 & \begin{tabular}{|c|}
10.43 \\
$(3.3) \mathrm{bc}$
\end{tabular} & $\begin{array}{c}10.61 \\
(3.32) \mathrm{bc}\end{array}$ & $\begin{array}{c}9.63 \\
(3.17) \mathrm{bcd}\end{array}$ & \begin{tabular}{|c|}
8.89 \\
$(3.06) \mathrm{bc}$
\end{tabular} & $\mid \begin{array}{c}9.89 \\
(3.22) \mathrm{bc}\end{array}$ & 54.18 & $\begin{array}{c}8.24 \\
(2.95) \mathrm{bcd}\end{array}$ & \begin{tabular}{|c|}
7.48 \\
$(2.82) \mathrm{b}$
\end{tabular} & $\begin{array}{c}5.99 \\
(2.55) \mathrm{bc}\end{array}$ & \begin{tabular}{|c|}
4.71 \\
$(2.28) \mathrm{bc}$
\end{tabular} & $\begin{array}{c}6.60 \\
(2.67) \mathrm{bc}\end{array}$ & 70.85 & \begin{tabular}{|c|}
10.17 \\
$(3.23) \mathrm{bc}$
\end{tabular} & 52.14 \\
\hline $\mathrm{T}_{8}$ & $\begin{array}{l}18.50 \\
(4.35)\end{array}$ & $\begin{array}{c}13.45 \\
(3.73) \mathrm{ab}\end{array}$ & $\begin{array}{c}11.15 \\
(3.41) \mathrm{ab}\end{array}$ & $\begin{array}{c}9.24 \\
(3.11) \mathrm{ab} \\
(\end{array}$ & $\begin{array}{c}13.08 \\
(3.67) \mathrm{ab}\end{array} \mid 3$ & 32.89 & \begin{tabular}{|c|}
9.46 \\
$(3.15) \mathrm{b}$
\end{tabular} & $\begin{array}{c}9.23 \\
(3.07) \mathrm{b}\end{array}$ & $\begin{array}{c}8.91 \\
(3.06) \mathrm{b}\end{array}$ & \begin{tabular}{|c|}
8.44 \\
$(2.99) \mathrm{a}$
\end{tabular} & $\begin{array}{c}9.01 \\
(3.08) \mathrm{b}\end{array}$ & 58.25 & $\begin{array}{c}7.93 \\
(2.88) \mathrm{bc}\end{array}$ & \begin{tabular}{|c|}
7.46 \\
$(2.82) \mathrm{b}$
\end{tabular} & $\begin{array}{c}4.89 \\
(2.32) \mathrm{b}\end{array}$ & $\begin{array}{c}3.53 \\
(2.00) \mathrm{b}\end{array}$ & $\begin{array}{c}5.95 \\
(2.54) \mathrm{b}\end{array}$ & 73.72 & $\begin{array}{c}9.35 \\
(3.1) \mathrm{b}\end{array}$ & 55.99 \\
\hline $\mathrm{T}_{9}$ & $\begin{array}{l}18.48 \\
(4.35)\end{array}$ & $\begin{array}{c}19.29 \\
(4.39) \mathrm{b}\end{array}$ & \begin{tabular}{c|}
19.85 \\
$(4.47) \mathrm{c}$
\end{tabular} & $\begin{array}{c}21.06 \\
(4.56) \mathrm{b}\end{array}$ & $\begin{array}{c}19.50 \\
(4.46) \mathrm{b}\end{array}$ & 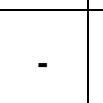 & \begin{tabular}{|c|}
21.51 \\
$(4.68) \mathrm{c}$
\end{tabular} & \begin{tabular}{|c|}
21.18 \\
$(4.65) \mathrm{c}$
\end{tabular} & \begin{tabular}{c|}
21.81 \\
$(4.72) \mathrm{e}$
\end{tabular} & \begin{tabular}{c|}
21.85 \\
$(4.72) \mathrm{c}$
\end{tabular} & \begin{tabular}{|c|}
21.59 \\
$(4.62) \mathrm{c}$
\end{tabular} & & $\begin{array}{c}22.45 \\
(4.73) \mathrm{d}\end{array}$ & $\begin{array}{c}22.61 \\
(4.77) \mathrm{d}\end{array}$ & $\begin{array}{c}21.69 \\
(4.65) \mathrm{f}\end{array}$ & $\begin{array}{c}23.14 \\
(4.83) \mathrm{e}\end{array}$ & $\begin{array}{c}22.65 \\
(4.75) \mathrm{e}\end{array}$ & - & $\begin{array}{c}21.24 \\
(4.64) \mathrm{d}\end{array}$ & \\
\hline S.Em. \pm & & 0.07 & \begin{tabular}{l|l}
0.05 &
\end{tabular} & \begin{tabular}{l|l}
0.07 \\
\end{tabular} & 0.03 & & \begin{tabular}{|l|}
0.04 \\
\end{tabular} & 0.04 & \begin{tabular}{l|l}
0.02 &
\end{tabular} & 0.02 & 0.04 & & 0.05 & 0.03 & 0.05 & 0.03 & 0.04 & & 0.05 & \\
\hline C.D. (0.05) & NS & 0.20 & 0.14 & 0.20 & 0.09 & & 0.11 & 0.13 & 0.06 & 0.06 & 0.12 & & 0.16 & 0.10 & 0.14 & 0.09 & 0.11 & & 0.14 & \\
\hline $\mathrm{CV}(\%)$ & & 11.32 & 10.14 & 12.29 & 7.61 & & 9.22 & 10.26 & 7.08 & 7.17 & 9.91 & & 11.78 & 9.96 & 12.53 & 10.54 & 10.87 & & 10.54 & \\
\hline
\end{tabular}

$\mathrm{T}_{1}$-Profenophos $50 \mathrm{EC} @ 2 \mathrm{~mL} / \mathrm{L}, \mathrm{T}_{2}$ - Novaluron 10EC (Rimon 10EC) @ $1.0 \mathrm{~mL} / \mathrm{L}, \mathrm{T}_{3}-$ Azadirichtin1500ppm @ $5 \mathrm{~mL} / \mathrm{L}, \mathrm{T}_{4}-\mathrm{Thiodicarb} 70 \mathrm{WP} @ 1.0 \mathrm{~g} / \mathrm{ml}$,

$\mathrm{T}_{5}$ - Profenophos 50EC @ $2 \mathrm{~mL} / \mathrm{L}+\mathrm{DDVP} 76 \mathrm{EC} @ 0.5 \mathrm{~mL} / \mathrm{L}, \mathrm{T}_{6}-$ Novaluron 10EC @ $1.0 \mathrm{~mL} / \mathrm{L}+\mathrm{DDVP} 76 \mathrm{EC} @ 0.5 \mathrm{~mL} / \mathrm{L}$,

$\mathrm{T}_{7}$ - Azadirichtin 1500ppm @ $5 \mathrm{~mL} / \mathrm{L}+$ DDVP 76EC @ $0.5 \mathrm{~mL} / \mathrm{L}, \mathrm{T}_{8}$ - Thiodicarb 70WP @ 1 $/ \mathrm{L}+$ DDVP 76EC @ $0.5 \mathrm{~mL} / \mathrm{L}, \mathrm{T}_{9}-\mathrm{Untreated}$ control *BS: Before spraying, $* *$ DAS: Days after spraying, ***POC: Per cent reduction over control,

****: Values in parentheses are $\sqrt{ } \mathrm{x}+0.5$ transformed value, Means followed by common alphabet do not differ significantly 
Table.2 Bio efficacy of insecticides on grub load, tunnel length and broken branches caused by the shoot weevil infestation in okra

\begin{tabular}{|c|c|c|c|c|}
\hline Treatment details & $\begin{array}{c}\text { No of galls per } \\
\text { plant (Mean from } \\
10 \text { infested plants) }\end{array}$ & $\begin{array}{c}\text { No of broken } \\
\text { branches per } 10 \\
\text { infested plants }\end{array}$ & $\begin{array}{l}\text { No of grubs per } \\
\text { plant (Mean from } \\
10 \text { infested plants) }\end{array}$ & $\begin{array}{l}\text { Average tunnel } \\
\text { length }(\mathrm{cm}) \text { from } \\
10 \text { infested plants }\end{array}$ \\
\hline $\mathrm{T}_{1}$-Profenophos $50 \mathrm{EC} @ 2 \mathrm{~mL} / \mathrm{L}$ & $\begin{array}{c}5.07 \\
(2.35) \mathrm{bcd}\end{array}$ & $\begin{array}{c}3.53 \\
(2.01) \mathrm{bcd}\end{array}$ & $\begin{array}{c}2.93 \\
(1.85) \mathrm{bc}\end{array}$ & $\begin{array}{c}11.71 \\
(3.49) \mathrm{bc}\end{array}$ \\
\hline $\mathrm{T}_{2}-$ Novaluron 10EC (Rimon 10EC) @ $1.0 \mathrm{~mL} / \mathrm{L}$ & $\begin{array}{c}6.07 \\
(2.56) \mathrm{de}\end{array}$ & $\begin{array}{c}5.87 \\
(2.52) \mathrm{d}\end{array}$ & $\begin{array}{c}3.8 \\
(2.07) \mathrm{bc}\end{array}$ & $\begin{array}{c}15.07 \\
(3.91) \mathrm{bc}\end{array}$ \\
\hline $\mathrm{T}_{3}-$ Azadirichtin 1500ppm @ $5 \mathrm{~mL} / \mathrm{L}$ & $\begin{array}{c}7.5 \\
(2.83) \mathrm{ef}\end{array}$ & $\begin{array}{c}5.12 \\
(2.37) \mathrm{cd}\end{array}$ & $\begin{array}{c}4.37 \\
(2.21) \mathrm{cd}\end{array}$ & $\begin{array}{l}15.65 \\
(4.01) \mathrm{c}\end{array}$ \\
\hline $\mathrm{T}_{4}-$ Thiodicarb 70 WP @ $1.0 \mathrm{~g} / \mathrm{L}$ & $\begin{array}{c}5.53 \\
(2.45) \mathrm{cd}\end{array}$ & $\begin{array}{c}3.87 \\
(2.09) \mathrm{bcd}\end{array}$ & $\begin{array}{c}3.73 \\
(2.05) \mathrm{bc}\end{array}$ & $\begin{array}{c}12.07 \\
(3.54) \mathrm{bc}\end{array}$ \\
\hline $\begin{array}{l}\mathrm{T}_{5}-\text { Profenophos } 50 \text { EC@ } 2 \text { mL/L +DDVP } 76 \text { EC @ } 0.5 \\
\text { mL/L }\end{array}$ & $\begin{array}{c}1.23 \\
(1.25) \mathrm{a}\end{array}$ & $\begin{array}{c}1.00 \\
(1.18) \mathrm{a}\end{array}$ & $\begin{array}{c}0.87 \\
(1.06) \mathrm{a}\end{array}$ & $\begin{array}{c}7.3 \\
(2.78) \mathrm{a}\end{array}$ \\
\hline $\begin{array}{l}\mathrm{T}_{6}-\text { Novaluron 10EC @ } 1.0 \text { mL/L+ DDVP 76 EC @ } 0.5 \\
\mathrm{~mL} / \mathrm{L}\end{array}$ & $\begin{array}{c}3.6 \\
(2.0) \mathrm{abc}\end{array}$ & $\begin{array}{c}2.4 \\
(1.69) \mathrm{ab}\end{array}$ & $\begin{array}{c}2.53 \\
(1.74) b\end{array}$ & $\begin{array}{c}9.9 \\
(3.18) \mathrm{b}\end{array}$ \\
\hline $\begin{array}{l}\mathrm{T}_{7} \text { - Azadirichtin 1500ppm @ } 5 \mathrm{~mL} / \mathrm{L}+\text { DDVP76EC @ } 0.5 \\
\mathrm{~mL} / \mathrm{L}\end{array}$ & $\begin{array}{c}4 \\
(2.11) \mathrm{bcd} \\
\end{array}$ & $\begin{array}{c}3.47 \\
(1.99) \mathrm{bc}\end{array}$ & $\begin{array}{c}2.6 \\
(1.74) b\end{array}$ & $\begin{array}{l}10.19 \\
(3.26) \mathrm{bc}\end{array}$ \\
\hline $\begin{array}{l}\mathrm{T}_{8}-\text { Thiodicarb } 70 \text { WP@ } 1.0 \mathrm{~g} / \mathrm{L}+\mathrm{DDVP} 76 \mathrm{EC} @ 0.5 \\
\mathrm{~mL} / \mathrm{L}\end{array}$ & $\begin{array}{c}2 \\
(1.58) \mathrm{ab}\end{array}$ & $\begin{array}{c}2.1 \\
(1.61) \mathrm{a}\end{array}$ & $\begin{array}{c}1.80 \\
(1.5) \mathrm{b}\end{array}$ & $\begin{array}{c}8.73 \\
(3.01) \mathrm{b}\end{array}$ \\
\hline $\mathrm{T}_{9}-$ Untreated control & $\begin{array}{c}8.63 \\
(3.02) f\end{array}$ & $\begin{array}{c}6.47 \\
(2.64) \mathrm{e}\end{array}$ & $\begin{array}{c}6.82 \\
(2.7) \mathrm{d}\end{array}$ & $\begin{array}{c}23.87 \\
(4.93) \mathrm{c}\end{array}$ \\
\hline S.Em. \pm & 0.03 & 0.02 & 0.02 & 0.06 \\
\hline $\mathrm{CD}(\mathrm{P}=0.05)$ & 0.08 & 0.05 & 0.07 & 0.19 \\
\hline $\mathrm{CV}(\%)$ & 12.29 & 10.86 & 14.07 & 12.28 \\
\hline
\end{tabular}

Figures in parentheses are $\sqrt{ } x+0.5$ transformation values, DAS - Days after sowing

Means followed by common alphabet do not differ significant 
Table. 3 Cost economics of shoot weevil management with different insecticides in okra

\begin{tabular}{|c|c|c|c|c|c|c|c|c|}
\hline Treatment details & $\begin{array}{c}\text { Cost of } \\
\text { cultivation } \\
\text { (Rs./ha) }\end{array}$ & $\begin{array}{c}\text { Cost of } \\
\text { plant } \\
\text { protection } \\
\text { (Rs./ha) }\end{array}$ & $\begin{array}{l}\text { Total cost of } \\
\text { production } \\
\text { (Rs./ha) }\end{array}$ & $\begin{array}{l}\text { Yield (kg } \\
\quad \text { /ha) }\end{array}$ & $\begin{array}{c}\text { Per cent } \\
\text { increase } \\
\text { in yield } \\
\text { over } \\
\text { control }\end{array}$ & $\begin{array}{c}\text { Gross } \\
\text { returns } \\
\text { (Rs./ha) }\end{array}$ & $\begin{array}{l}\text { Net } \\
\text { returns } \\
\text { (Rs./ha) }\end{array}$ & B:C \\
\hline $\mathrm{T}_{1}$-Profenophos 50 EC @ $2 \mathrm{~mL} / \mathrm{L}$ & 15000 & 5400 & 20400 & 6098.40 & 79.25 & 60984.00 & 40584.00 & 2.98 \\
\hline $\mathrm{T}_{2}$ - Novaluron 10EC (Rimon 10EC) @ $1.0 \mathrm{~mL} / \mathrm{L}$ & 15000 & 11616 & 26616 & 5600.00 & 77.42 & 56000.00 & 29384.00 & 2.10 \\
\hline $\mathrm{T}_{3}-$ Azadirichtin 1500ppm @ $5 \mathrm{~mL} / \mathrm{L}$ & 15000 & 5880 & 20880 & 5176.889 & 75.57 & 51768.89 & 30888.89 & 2.47 \\
\hline $\mathrm{T}_{4}-$ Thiodicarb 70 WP @ $1.0 \mathrm{~g} / \mathrm{ml}$ & 15000 & 7680 & 22680 & 5712.00 & 77.86 & 57120.00 & 34440.00 & 2.51 \\
\hline $\begin{array}{l}\mathrm{T}_{5}-\text { Profenophos 50EC @ } 2 \text { mL/L+DDVP } 76 \text { EC @ } 0.5 \\
\mathrm{~mL} / \mathrm{L}\end{array}$ & 15000 & 6700 & 21700 & 8316.933 & 84.79 & 83169.33 & 61469.33 & 3.83 \\
\hline $\begin{array}{l}\mathrm{T}_{6}-\text { Novaluron 10EC @ } 1.0 \text { mL/L + DDVP } 76 \text { EC @ } 0.5 \\
\mathrm{~mL} / \mathrm{L}\end{array}$ & 15000 & 12916 & 27916 & 6485.333 & 80.50 & 64853.33 & 36937.33 & 2.32 \\
\hline $\begin{array}{l}\mathrm{T}_{7} \text { - Azadirichtin 1500ppm @ } 5 \text { mL/L+DDVP76EC @ } 0.5 \\
\mathrm{~mL} / \mathrm{L}\end{array}$ & 15000 & 7180 & 22180 & 6048.00 & 79.09 & 60480.00 & 38300.00 & 2.72 \\
\hline $\begin{array}{l}\mathrm{T}_{8}-\text { Thiodicarb } 70 \text { WP @ } 1.0 \mathrm{~g} / \mathrm{L}+\text { DDVP 76EC @ } 0.5 \\
\mathrm{~mL} / \mathrm{L}\end{array}$ & 15000 & 8980 & 23980 & 6850.133 & 81.54 & 68501.33 & 44521.33 & 2.85 \\
\hline $\mathrm{T}_{9}-$ Untreated control & 15000 & 0 & 15000 & 1264.28 & - & 12642.80 & -2357.20 & 0.842 \\
\hline
\end{tabular}

* - Totally three spray are taken at 20, 40 and 60 DAS in all the treatments, Market price of okra fruit at harvestin period (2015) : 10 Rs./kg,

Market price of chemicals used in experiment (2015)

Profenophos 50 EC - Rs 900/Ltr,

DDVP 76 E - Rs 650/ Ltr,

Novuluron (Rimon 10 EC) - Rs 968 / 250 ml,

Azadirichtin - Rs 420/ Ltr, Thiodicarb 70 WP - Rs 1280 / 500 g 
The results revealed significant variation in okra fruit yield among the different treatments. Significantly highest of 8316.93 $\mathrm{kg} / \mathrm{ha}$ with higher increase of 84.79 per cent in okra fruit yield was recorded in application of Profenophos50 EC @ $2 \mathrm{~mL} / \mathrm{L}$ + DDVP 76E@0.5 mL/L, followed by Thiodicarb 70WP @ 1.0g/L + DDVP 76 EC @ 0.5 mL/L (6850.13 kg/ha with $81.54 \%$ increase) (Table $3)$. Highest benefit cost ratio (B: $\mathrm{C}$ ratio) of 3.83 was accounted in Profenophos 50 EC @ $2 \mathrm{~mL} / \mathrm{L}+$ DDVP 76EC @ $0.5 \mathrm{~mL} / \mathrm{L}$ followed by 2.85 in Thiodicarb 70WP@1.0g/L + DDVP 76EC@0.5 mL/L. The lowest B: C of 0.842 was recorded in untreated control (Table 3).

The information on combined action of Profenophos 50 EC + DDVP 76EC and Thiodicarb 70WP + DDVP 76EC against shoot weevil is being reported for the first time on okra. The similar studies on the shoot weevil in okra are lacking to compare the present findings. However, Ayyanna (2012) reported two sprays at 30 and 50 DAS of Thiodicarb 70WP @ 1g/L + DDVP 76EC @ $1 \mathrm{~mL} / \mathrm{L}$, Profenophos 50 EC @ $2 \mathrm{~mL} / \mathrm{L}+$ DDVP 76EC @ $1 \mathrm{~mL} / \mathrm{L}$ and Thiodicarb 70WP@1g/L alone were found to be effective against shoot weevil, A. Affaber. Further, Ganesha halikatti (2015) reported spraying with Profenophos 50 EC @ 2 mL/L at 30 and 45 DAS recorded lower incidence of shoot weevil and highest net returns in $B t$ cotton but single chemical intervention followed at various stages of crop was found to be ineffective for reducing the infestation upto the mark.

Present study revealed that insecticides such as Profenophos 50EC, Novaluron 10EC, Thiodicarb 70WP in combination with DDVP 76EC were very effective against $A$. affaber on okra with reduced incidence in number of adults, per cent grub infestation, grub load, enhanced okra fruit yield and higher benefit cost ratio.
Basically, both profenophos and thiodicarb possess the ovicidal and larvicidal characteristics and contact in action, the application of these insecticides certainly suppressed both egg as well as grub stages of shoot weevil at the early stages, eggs that are laid at the tip of the shoot get killed due to ovicidal action of insecticide. Which resulted in effective reduction of shoot weevil infestation. Further, mixing of DDVP with these two insecticides enhanced the efficacy of their combination owing to fumigant and penetrating nature of DDVP.

Reduction in the stem tunnel length over control was noticed in treatments having the spray chemical in combination with DDVP 76EC this may be due to immediate killing of grubs through action of DDVP inside stem may be due to optimum administration of chemical in the grub entrance hole which inferred good mortality of weevil without any phytotoxic effect on plants.

The adult females of shoot weevil alights on the cotton shoots for making ovipositional scoops to lay eggs by inserting its snout. The pre tarsal segments of the weevils come in contact with insecticide treated surface on shoot tip. Since the weevils takes longer time for scooping and inserting eggs, the weevils severely get affected by contact action of insecticides that inhibits the activity of neurotransmitter acetylcholine, which plays an important role in regulating the function of brain, heart and skeletal muscles, hence the activity pauses by inhabiting the acetylcholine esterase enzyme in synapse due to over accumulation of acetylcholine by opening of $\mathrm{Na}^{++}$and $\mathrm{K}^{+}$channels that leads to death of weevils due to respiratory failure.

The lower grub and adult population was also recorded in Azadirichtin 1500ppm in combination with DDVP 76EC sprayed treatment as compared to chemicals without combination with DDVP 76EC which might 
be due to phagodeterant effect of Nimbecidine and fumigant as well as penetrating action of DDVP. However, the higher adult and grub infestation as compared to superior treatments recorded in the treatment having spray with Nimbecidine alone might be due to slower and nonpenetrating action of insecticide which failed to mitigate the infestation.

\section{References}

Anonymous, 2015. Horticulture Statistics 2015-16, Govt. Of India.

Aurivillius, C., 1891. Collection d' insects' formaedans I Indo-China par M. Pavic, Consul-de France all Cambodege. Coleopteres, Curculionies. Nour. Archs. Mus. Hist. nat. Paris, 3 (3): 205-244.

Ayyanna, B., 2012. Population dynamics and management of shoot weevil, Alcidodes affaber Aurivillius, on Bt cotton. M. Sc. (Agri.) Thesis, Univ. Agric. Sci.,
Dharwad, Karnataka (India).

Azadirichtin, T. V. R., 1922. The weevil fauna of South India with special references to the species of economic importance, Agric. Res. Inst. Pusa, Bull., 125: 21.

Deshpande, V. P., 1990. Investigation on cotton shoot weevil, Alcidodes affaber Aurivillius (coleoptera: curculionidae) Ph. D. Thesis, Uni. Agric. Sci., Dharwad, Karnataka (India).

Ganesha Halikatti, 2015. Studies on biology and management of shoot weevil, Alcidodes affaber Aurivillus in $\mathrm{Bt}$ Cotton. Ph. D. Thesis, Univ. Agric. Sci., Dharwad, Karnataka (India).

Srinivasa Rao, and Rajendran, R., 2003. Joint action potential of neem with other plant extracts against the leaf hopper Amrasca devastance (Distant) on Okra. Pest Mangt. And Econ. Zool., 10: 131136.

\section{How to cite this article:}

Manjunatha, H.A., S.B. Patil, S.S. Udikeri and Shamarao Jahagirdhar. 2017. Study on Bioefficacy of Insecticides against Shoot Weevil (Alcidodes affaber Aurivillius) in Okra (Abelmoschus esculentus Linn.). Int.J.Curr.Microbiol.App.Sci. 6(9): 3447-3456. doi: https://doi.org/10.20546/ijcmas.2017.609.423 